\title{
El CDS como plataforma de la diplomacia de defensa Argentina (2008-2015)
}

\section{The South American Defense Council as a platform for Argentine defense diplomacy (2008-2015)}

Rev. Bras. Est. Def. v. 4, no 2, jul./dez. 2017, p. 175-197

DOI: 10.26792/RBED.v4n2.2017.74981

ISSN 2358-3932

SOL GASTALDI

\section{CONSIDERACIONES PRELIMINARES Y ANTECEDENTES HISTÓRICOS}

La "diplomacia de la defensa" como práctica gubernamental no es nueva en Argentina. La novedad es que la misma se desarrolla en el marco del gobierno civil de la defensa. ${ }^{1}$ Antes de la creación en el año 2007 de la Secretaría de Asuntos Internacionales para la Defensa del Ministerio de Defensa y de la implementación del Ciclo de Planeamiento, la mayor parte de la cooperación internacional se desarrollaba a expensas del control civil, por iniciativa propia de las FFAA y sin la debida articulación con la Cancillería. Las FFAA se constituían como agentes de una "diplomacia militar" que buscaba incrementar la cooperación castrense con otras FFAA, lo que implicaba que, en ocasiones, esta cooperación podía no corresponderse con los objetivos políticos de la jurisdicción.

Así, como parte de la tendencia de impulsar la diplomacia militar, en el ámbito hemisférico se crearon organismos de cooperación multilateral entre FFAA, como la Conferencia de Ejércitos Americanos (CEA) en 1960. Por esos años también tuvieron su origen la Conferencia Naval Interamericana (CNI) y el Sistema de Cooperación de las Fuerzas Aéreas Americanas (Sicofaa). Argentina participa desde esos años de estos mecanismos, los cuales se han caracterizado por la hegemonía político-militar de los Estados Unidos y la proyección de sus intereses hacia Latinoamérica.

La CEA se fundó en plena Guerra Fría por iniciativa de los Estados Unidos con el propósito de impulsar la "reorientación de las preocupaciones defensivas continentales desde la agresión externa hacia la subversión interna, desde el enemigo militar clásico, hacia el enemigo ideológico, el

Sol Gastaldi - Universidad de Buenos Aires / Universidad de la Defensa Nacional. 
infiltrado comunista y la agitación guerrillera” (Caviasca 2015, 18). Como plataforma para la implementación en los países latinoamericanos de la Doctrina de la Seguridad Nacional (DSN), impulsó cambios doctrinarios que modificaron las hipótesis de empleo del instrumento militar, y por tanto, el esquema de equipamiento, adiestramiento y despliegue para avanzar en el combate del enemigo interno (Caviasca 2015).

La CNI, por su parte, nació en 1959 como foro internacional que nuclea a los titulares de las fuerzas navales del continente americano con el fin de propiciar el intercambio de ideas, experiencias y conocimientos sobre los problemas comunes que afectan a las armadas o marinas del hemisferio, establecer contactos permanentes y promover la confianza mutua. Con una meta afín, el Sicofaa fue fundado en 1961. En su primera Carta Constitutiva, se expresaba que la cooperación entre las fuerzas aéreas americanas se materializaba a través de una serie de funciones básicas como apoyo logístico, búsqueda y rescate, apoyo en caso de desastre, instrucción, acción cívica, contrainsurgencia, entre otras, expresando la dinámica que tenían estas instituciones militares de cooperación en el marco de la DSN. De acuerdo a la evolución de la situación estratégica hemisférica y de las amenazas que enfrenta el continente, dichas funciones fueron cambiando al presente.

Contrariamente a estas iniciativas de diplomacia militar, se destaca la creación en 2008 del Consejo de Defensa Suramericano (CDS), que se constituye como una iniciativa inédita de cooperación regional en materia de defensa por dos cuestiones: primero, porque se conformó como una instancia estrictamente sudamericana, sin la intervención o participación de los Estados Unidos; segundo, porque los políticos elegidos democráticamente eran los representantes de los países en el organismo, en lugar de los altos mandos militares.

Todos los organismos antes mencionados se constituyen en plataformas para la promoción de los intereses nacionales de los países participantes desde una perspectiva cooperativa; en virtud de ello, conforman un punto de encuentro entre la política de defensa y la política exterior. Tal como sostiene Azuara Blanco (2009, 75), "diplomacia y defensa son las dos caras de la política exterior de un Estado”.

\section{LA DIPLOMACIA DE LA DEFENSA: ELEMENTOS CONCEPTUALES}

Si bien algunos autores coinciden en señalar que el origen del desarrollo conceptual de la diplomacia de la defensa remite al abordaje que el Reino Unido realizó a la cuestión en la Revisión Estratégica de 1998, la diplomacia de la defensa no es una práctica gubernamental novedosa. Así lo entiende Azuara Blanco (2009, 92), al advertir que en la actualidad 
"la expresión "diplomacia de la defensa" engloba un amplio abanico de actividades que en el pasado han sido definidas como cooperación militar o asistencia militar". Tal es el caso de las funciones de los agregados de defensa en las embajadas o agregadurías militares en el exterior, que datan del siglo XIX. A pesar de ello, Azuara Blanco considera que lo novedoso es el enfoque conceptual que se le otorga a ésta, al reconocerla como una actividad contributiva al campo de la prevención de conflictos.

En este marco, Azuara Blanco (2009, 93) define a la diplomacia de la defensa como "el conjunto de actividades no operacionales que ejecutan las organizaciones y unidades dependientes del Ministerio de Defensa en beneficio de la diplomacia tradicional, para la prevención de conflictos, para el establecimiento de medidas de confianza y para el apoyo al desarrollo armónico de FFAA de terceros países, en beneficio del propio Estado o de organizaciones supranacionales a las que se halle adscrito".

Por su parte, Gregory Winger (2014) identifica a la diplomacia de la defensa como una variante de poder blando, que se emplea para cooptar el pensamiento estratégico de otro Estado. A partir de tal hipótesis, Winger afirma que la vinculación de la diplomacia de defensa con el concepto de poder blando deviene en una herramienta geopolítica efectiva. En efecto, como señala Winger, mientras el vínculo entre las FFAA y el poder duro resulta claramente observable, el uso del aparato de defensa de un Estado como fuente de poder blando permanece como un ámbito analíticamente poco explorado. Siguiendo este pensamiento, Arancibia Morales (2013) agrega que la diplomacia de la defensa, como instrumento de política exterior, refleja "un smart power" que combina atributos convencionales - hard power - con otros culturales y políticos - soft power.

Azuara Blanco (2009) agrega que, en apoyo a la política exterior, la diplomacia de defensa procura establecer cierta influencia político-militar sobre otros Estados o en la arena internacional.

Dado que el propósito de este trabajo no es profundizar en tales debates teóricos, sino alcanzar una aproximación conceptual que permita analizar la diplomacia de la defensa en Argentina, adoptaremos la definición que toma el Ministerio de Defensa español (España, 2011), que considera a la misma como el conjunto de actividades internacionales basadas principalmente en el diálogo y la cooperación que realiza el Ministerio de Defensa a nivel bilateral con sus aliados, socios y países amigos para impulsar el cumplimiento de los objetivos de la política de defensa en apoyo de la acción exterior del Estado.

De esta manera, la definición recogida aquí nos permitirá avanzar en la diferenciación teórica de la diplomacia de la defensa de otro concepto que, si bien suelen emplearse en forma análoga, resulta relevante con- 
siderar a los fines del estudio del caso argentino. Tal concepto es el de "diplomacia militar".

La diplomacia militar refiere a las relaciones de cooperación entre FFAA de distintos países. Como práctica, remite a la diplomacia europea del siglo XIX, que se articulaba principalmente alrededor de las actividades de los agregados de defensa, mencionados como diplomáticos de uniforme (Swistek 2012). Siguiendo a Swistek (2012) y a Cottey y Foster (2004), el rol tradicional de las FFAA se definía de acuerdo a su capacidad y preparación para usar la fuerza y enfrentar amenazas estatales militares externas con fines de defensa, disuasión o intervención. Pero, con el fin de conflicto bipolar, la diplomacia militar empezó a concentrarse en la cooperación, resultando así en un instrumento para la construcción de confianza entre las FFAA de los Estados.

En forma esquemática, pueden detallarse a las siguientes actividades como pertenecientes a la esfera de la diplomacia de la defensa:

1. Medidas de fomento de la confianza y la seguridad (bilaterales y multilaterales), como acuerdos de defensa; reuniones; intercambios y visitas recíprocas de alto nivel; mecanismos de transparencia y control de armamentos; ejercicios combinados; fuerzas combinadas;

2. Representaciones oficiales permanentes;

3. Entrenamiento de personal militar y formación de profesionales (civiles y militares) en el extranjero;

4. Participación activa en foros internacionales de seguridad y defensa; y

5. Cooperación industrial y tecnológica, como desarrollos con otros países de material y equipamiento militar.

Como puede observarse, algunas de estas actividades caen también dentro de la tradicional órbita de la diplomacia militar. Entonces, ¿cómo podemos establecer los puntos distintivos entre ambas?

En primer lugar, debemos señalar que la diplomacia militar constituye un componente de la diplomacia de la defensa, aunque pueden desarrollarse de manera autónoma. Dicha autonomía o concordancia derivarán del modelo de control civil sobre las FFAA. En aquellos países donde se desarrolle la conducción política de la jurisdicción, es decir, bajo un modelo de efectivo gobierno civil, plenamente institucionalizado, la diplomacia militar se articulará racionalmente con la diplomacia de la defensa. Contrariamente, cuando exista un déficit de control civil, o en modelos de tipo delegativos de la conducción civil en las FFAA (Gastaldi 2013) ambas esferas pueden distanciarse, pudiendo incluso no existir una diplomacia de la defensa. 
En segundo lugar, existe una diferencia en cuanto a los actores intervinientes. Mientras la diplomacia militar refiere a las acciones de cooperación castrense que emprenden las FFAA, la diplomacia de la defensa abarca al conjunto del sistema de defensa nacional.

En tercer lugar, la diplomacia de la defensa se constituye como la dimensión internacional de la política de Defensa (Eissa 2013). Sus objetivos, lineamientos y acciones se derivan del mayor nivel de conducción, es decir, del estratégico nacional. Por su parte, la diplomacia militar responde a un nivel estratégico militar y operacional.

La diplomacia de la defensa como herramienta de gestión pública contribuye entonces en forma directa a la política exterior de la Nación. En tal sentido, la existencia de mecanismos interinstitucionales entre ambas esferas determinará la capacidad de la diplomacia de la defensa de concurrir en forma proactiva y asertiva a ésta. Dicho esto, la coordinación entre ambas provee una "vinculación directa y lineal entre la posición que un Estado ocupa en el sistema internacional, las metas que desea lograr en la comunidad de naciones, sus objetivos estratégicos y el desarrollo y la mantención de las capacidades militares necesarias para cumplir dichos objetivos" (Navarro Meza 2009, 63. Citado por Arancibia Morales 2013, 7).

Como veremos a continuación, en el caso de Argentina, con la conformación de la Secretaría de Asuntos Internacionales de la Defensa en el 2007, se le otorgó a la diplomacia de la defensa mayor preeminencia y un rol clave en el desarrollo de la jurisdicción, en forma paralela al proceso de fortalecimiento del principio de conducción civil sobre las FFAA.

Asimismo, a través de la creación del Ciclo de Planeamiento en el mismo año, se estableció un mecanismo formal de articulación entre la política exterior y la política de defensa nacional, a través del cual se establecen racionalmente los medios y fines que derivarán en un modelo militar adecuado al logro de los objetivos nacionales plasmados.

\section{LA DIMENSIÓN INTERNACIONAL DE LA POLÍTICA DE DEFENSA}

A partir del año 2007, el Ministerio de Defensa argentino se volcó a fortalecer la dimensión internacional de la política de defensa, a través del establecimiento de una dependencia ministerial responsable de entender en los asuntos de cooperación internacional, interviniendo a dicho fin en la negociación de acuerdos bilaterales y multilaterales inherentes a la defensa nacional, en la aprobación de los acuerdos celebrados por el instrumento militar con otras FFAA, en la planificación y seguimiento de la ejecución de ejercicios combinados y operaciones de paz en las que participen las FFAA argentinas, en la planificación y ejecución de políticas de contralor del material 
para la defensa y en la formulación de la posición del país en cuestiones internacionales de desarme. Así, mediante el Decreto $n^{\circ} 788 / 07$, la reestructuración que permitió la creación de la Secretaría de Asuntos Internacionales de la Defensa (SAID), junto a sus dos direcciones dependientes -Contralor de Material de Defensa y Cooperación para el Mantenimiento de la Paz- "se consumó a los fines de planificar y ejecutar de manera coherente y coordinada la vinculación política e institucional con Ministerios de Defensa, FFAA extranjeras y organismos multilaterales de defensa, así como optimizar y adecuar las actividades ministeriales y militares de cooperación internacional siguiendo los lineamientos determinados por el nivel de conducción estratégico nacional” (Ministerio de Defensa 2015b).

Este proceso fue acompañado por el establecimiento de mecanismos concretos de coordinación con la Cancillería. A tal efecto, se conformó en la SAID un área de política internacional responsable de la coordinación interministerial con el Ministerio de Relaciones Exteriores, Comercio Internacional y Culto, así como también de intervenir en los asuntos vinculados a la competencia del Ministerio de Defensa en materia de Política Antártica, que elabora Cancillería. ${ }^{1}$

En 2013, una reorganización de la orgánica ministerial dirigida a “perfeccionar el uso de los recursos públicos incrementando la calidad de la acción estatal”, según el texto del Decreto n 636/13, derivó en la conversión de la SAID en una Subsecretaría (SSAID), dependiente de la Secretaría de Estrategia y Asuntos Militares (SEAM). Esta decisión, que podría ser entendida como un empoderamiento de la SEAM y en consecuencia, de la dimensión estratégica de la política de defensa (Eissa 2013), fue acompañada al año siguiente por una nueva reestructuración al interior de la SSAID, ordenada a través del Decreto ${ }^{\circ} 1956 / 14$, creándose dos direcciones dentro de la Dirección General de Política Internacional de Defensa -Dirección de Asuntos Multilaterales y Dirección de Asuntos Bilaterales - y una Dirección de Agregadurías y Comisiones en el Exterior.

Según señala el Decreto citado, "el incremento de las relaciones bilaterales y los foros multilaterales de actuación, especialmente en el marco del Consejo de Defensa Suramericano(CDS) y su Centro de Estudios Estratégicos de Defensa, a lo que se suma cada dos (2) años la Conferencia de Ministros de Defensa de las Américas, la eventual concreción de la Conferencia de reestructuración del Sistema Interamericano de Defensa, la reunión de Ministros de Defensa y de Relaciones Exteriores de la Zona de Paz y Cooperación del Atlántico Sur, y otras reuniones bilaterales y multilaterales, requieren contar con equipos de trabajo y delegaciones suficientemente representativas para presentar y hacer valer las posturas nacionales en cada una de las referidas instancias". 


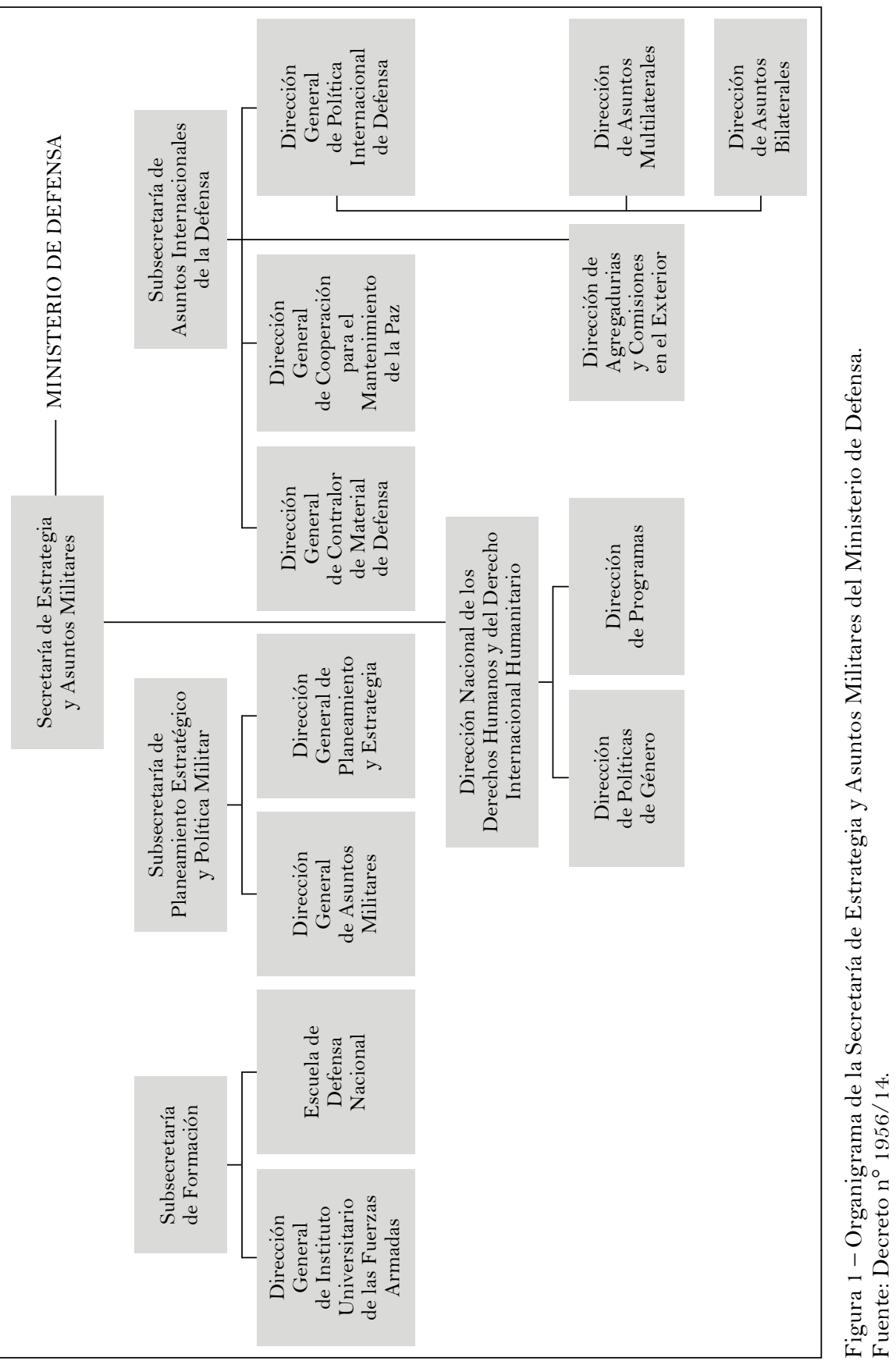


A partir de estas transformaciones, la dimensión internacional de la defensa se consolidó como un complemento de la política exterior del Estado, como herramienta para la proyección en el exterior de los valores de la política de defensa nacional y los intereses nacionales (Ministerio de Defensa 2010, 217).

En este marco, se encauzaron desde la SSAID diversas acciones, tales como la promoción del diálogo político-estratégico y el establecimiento de mecanismos institucionales permanentes con los Ministerios de Defensa y FFAA de los países de la región; la actualización de convenios bilaterales y el desarrollo de nuevos acuerdos de cooperación con países con los que no se registraban; la promoción de ejercicios militares combinados e intercambios entre FFAA, priorizando aquellos con los países del entorno sudamericano; el desarrollo de proyectos conjuntos de complementación científica, tecnológica y de producción para la defensa; la puesta en práctica de iniciativas de integración inéditas, como la conformación de la Fuerza de Paz Binacional Combinada argentino-chilena Cruz del Sur y la Compañía de Ingenieros Combinada peruano-argentina General San Martín; y el apoyo proactivo a la creación y consolidación del Consejo de Defensa Suramericano (Ministerio de Defensa 2010).

Otro aspecto a destacar es que la diplomacia de la defensa define áreas de priorización para el establecimiento y desarrollo de los relacionamientos internacionales, conforme con la política exterior. En tal sentido, "Suramérica representa el espacio prioritario para la presencia institucional y las acciones de cooperación y complementación en materia de defensa, seguida sucesivamente de América Latina, el continente americano y luego las regiones y actores relevantes a nuestros intereses en el resto del mundo" (Ministerio de Defensa 2010, 220). En base a ello se establecen los temas de mayor interés para direccionar la cooperación internacional con los países de la región, abarcando principalmente la educación e instrucción militar, formación de civiles en defensa; ciencia, tecnología y producción para la defensa; desarrollo y fortalecimiento de las capacidades militares; actividades antárticas, en conformidad con la política nacional antártica; el fomento de los Derechos Humanos, el Derecho Internacional Humanitario y el enfoque de género; el apoyo logístico y la protección civil y asistencia humanitaria en caso de desastres y catástrofes de origen natural o antrópico. En tanto, en el ámbito extra-regional, la diplomacia de defensa argentina se orienta principalmente hacia el mantenimiento de la paz y la seguridad internacional, apoyando a las Naciones Unidas y los regímenes de no proliferación y control de armas.

Así, la creación de la SAID permitió establecer un órgano ministerial responsable de orientar y adecuar la diplomacia militar a los criterios de 
cooperación internacional fijados por el Ejecutivo Nacional, permitiendo de tal modo el efectivo control civil sobre las actividades de cooperación, intercambios y asistencia desarrollados por las FFAA.

\section{EL CICLO DE PLANEAMIENTO DE LA DEFENSA NACIONAL Y LA DIPLOMACIA DE DEFENSA ARGENTINA}

En el 2007, en conformidad con la necesidad de rediseñar el Instrumento Militar según la concepción, posicionamiento y actitud estratégica de la República Argentina, se establece el Ciclo de Planeamiento de la Defensa Nacional (CPDN), a través del Decreto $n^{\circ} 1729 / 07$.

El CPDN constituye, según el espíritu de la norma, "el proceso esencial e insustituible del Sistema de Defensa Nacional en lo que refiere a la definición de todos los subsistemas del Instrumento Militar: recursos humanos, infraestructura, logística, material, información, adiestramiento, organización y doctrina”.

La arquitectura del mismo partió del reconocimiento del principio de conducción civil y procuró que en el proceso intervenga toda la jurisdicción Defensa, el Estado Mayor Conjunto y las FFAA. Para ello, se establece un procedimiento concatenado de producción de documentos que orientan, direccionan e instruyen desde el más alto nivel de conducción nacional, el diseño de fuerzas. De este modo, esta norma se enmarcó en un proceso de reorganización del sistema de defensa y de fortalecimiento del gobierno civil, mediante la institucionalización de procedimientos y el traspaso de competencias de la esfera militar al ámbito civil que se llevaron a cabo a partir del 2006, tras la reglamentación de la Ley de Defensa Nacional a través del Decreto $n^{\circ} 727$.

El CPDN se inicia con el dictado de una Directiva de Política de Defensa Nacional (DPDN) por parte del Presidente de la Nación a propuesta del Ministerio de Defensa. La DPDN es un documento de carácter sectorial en el cual se explicitan los lineamientos centrales de la política de defensa nacional y de la política militar y se establecen los criterios y parámetros bajo los cuales se determinará la organización, el funcionamiento, el empleo y la administración de las FFAA. De acuerdo al artículo $3^{\circ}$ del Decreto $\mathrm{n}^{\circ} 1.729 / 07$, la DPDN contiene tres capítulos: el primero, consiste en un diagnóstico y apreciación del escenario de defensa y seguridad global y regional en el que se identifican "de manera precisa las tendencias del mismo, los riesgos y las amenazas actuales a los intereses nacionales y, de ser factible, también los eventuales para el mediano y largo plazo". El segundo capítulo establece la concepción y posicionamiento estratégico nacional en materia de defensa y los lineamientos centrales de la política 
de defensa nacional. Finalmente, en el tercero, se fijan las directrices para la instrumentación de la política de defensa y su derivada política militar. Es en este último capítulo, donde el Nivel Estratégico Nacional -es decir, el mayor nivel de conducción política-determina las instrucciones para los órganos del Ministerio de Defensa, el Estado Mayor Conjunto y las FFAA.

La DPDN elaborada por el Ministerio de Defensa, antes de ser suscripta por el Presidente de la Nación, es revisada por la Cancillería. De esta forma, se confirma que la visión volcada por el Ministerio de Defensa en el primer capítulo de la DPDN se vincula y contribuye efectivamente a los objetivos de política exterior.

Una vez dictada la DPDN, a partir de los lineamientos y órdenes establecidos allí por el Nivel Estratégico Nacional, el Estado Mayor Conjunto inicia el planeamiento militar conjunto.

Esta herramienta de gestión surgida en el año 2007 posee por fin asegurar que el modelo de fuerzas, es decir, el diseño, organización y empleo del instrumento militar, sea funcional a los objetivos políticos.

En este marco, en el año 2009 se dictó la primera DPDN, que dio por inicio al primer ciclo de planeamiento. Éste culminó en el año 2011 con la elaboración del Plan de Capacidades Militares. En 2014, la Presidente de la Nación, Cristina Kirchner, suscribió una nueva Directiva que dio lugar al segundo ciclo de planeamiento con la firma del Decreto ${ }^{\circ} 2645$.

Es importante mencionar que el CPDN incorpora como metodología el planeamiento basado en capacidades. Dicha metodología resulta funcional en el marco estratégico de la Argentina, caracterizado por la progresiva eliminación de sus hipótesis de conflicto desde la recuperación de la democracia en 1983, y la consolidación de la zona de paz sudamericana. Bajo esta coyuntura, Argentina adopta una actitud y posicionamiento estratégico defensivo, asentado sobre un modelo autónomo y cooperativo; esto es, sin renunciar al derecho a la legítima defensa, procura y sostiene la cooperación con países amigos, entendiendo que la misma contribuye al fortalecimiento de las propias capacidades, a la consecución de los objetivos de la Defensa y a garantizar la paz y seguridad internacionales, tal como se plasma en el segundo capítulo de la DPDN. Tanto el carácter defensivo de la política de defensa, como su disposición cooperativa, en el marco del CPDN se convierten en criterios rectores para el diseño del Instrumento Militar.

Como puede observarse, la propia actitud estratégica en fomento de la cooperación, deviene en un modelo de fuerzas que se consustancia con los lineamientos de política exterior. Así, la DPDN establece un modelo cooperativo basado en círculos concéntricos, a través del cual se prioriza, en primer lugar, a América del Sur y el Atlántico Sur como espacio geoestratégico; luego a América Latina y el Caribe; y finalmente, al marco 
global, particularmente con aquellos países con los cuales existen oportunidades de complementariedad y cooperación, tales como los países del Grupo "BRICS” y el G-20, y aquellos que favorezcan el incremento de las relaciones Sur-Sur.

Asimismo, y siguiendo la letra del Decreto $n^{\circ}$ 2645/14, la dimensión internacional de la política de defensa adopta como objetivos generales la promoción y consolidación de América del Sur como zona de paz y la construcción progresiva de un Sistema de Defensa Regional y el mantenimiento de la paz y la seguridad internacionales.

En cuanto a las acciones a emprender en el marco de la diplomacia de la defensa, la DPDN establece en su tercer capítulo que se deberá incrementar "la presencia institucional de elementos del Sistema de Defensa Nacional en las regiones y países de interés prioritario, particularmente en la forma de agregadurías de Defensa, misiones y acuerdos de cooperación, e intercambios de formación y adiestramiento militar".

En tal sentido, las acciones de cooperación y complementación internacional, deberán priorizar, de acuerdo a la DPDN 2014, las siguientes áreas temáticas:

a. Mantenimiento de la paz y la seguridad internacional;

b. Formación y adiestramiento militar;

c. Capacitación de civiles para la Defensa;

d. Desarrollo de capacidades técnico-militares;

e. Planeamiento estratégico de la Defensa;

f. Doctrina combinada y conjunta;

g. Desarme y no proliferación;

h. Derechos Humanos, Derecho Internacional Humanitario y cuestiones de género;

i. Investigación científica, desarrollo tecnológico y producción para la Defensa;

j. Logística de material y apoyo logístico mutuo;

k. Actividades antárticas;

1. Apoyo a la comunidad en caso de desastres naturales y catástrofes.

A nivel político-institucional la DPDN establece como tarea consolidar la "planificación y supervisión de la interacción de carácter estratégico-militar y técnico-profesional del Estado Mayor Conjunto de las FFAA y de las FFAA con sus pares extranjeros. En consecuencia, el vínculo y accionar militar internacional de las FFAA se implementará en sintonía con las directivas que devienen de la política exterior y la Política de Defensa Nacional”. A tal efecto, se elaborarán los documentos orientativos nece- 
sarios para establecer criterios y procedimientos a seguir en materia de participación en instancias de consulta y diálogo multilateral y bilateral, informes de asistencia, selección de postulantes para agregadurías y comisiones al exterior, ejercicios combinados, entre otras.

Finalmente, la DPDN dispone que los acuerdos que firmen las FFAA "sólo tendrán validez en tanto sean ratificados por el Ministerio de Defensa”, reafirmando de este modo el principio de gobierno civil.

\section{EL CONSEJO DE DEFENSA SURAMERICANO COMO PLATAFORMA DE LA DIPLOMACIA DE LA DEFENSA ARGENTINA}

Durante los años noventa, la política exterior de Argentina se configuró en base a la liberalización de la economía siguiendo los fundamentos del denominado "Consenso de Washington". En consecuencia, la política de defensa se orientó en función del alineamiento estratégico con los Estados Unidos. ${ }^{3}$ Pero, la crisis del 2001 que sacudió al país dando muestras de la necesidad de salir de ese modelo económico, también evidenció la importancia de desarrollar a nivel internacional un esquema de inserción basado en una estrategia más autónoma, que acompañara la recuperación del aparato productivo y el empleo.

Paralelamente, en la región sudamericana, Centroamérica y El Caribe, Estados Unidos impulsó la incorporación de la agenda de las denominadas "nuevas amenazas" que buscó involucrar a las FFAA en el combate del narcotráfico, el terrorismo, el crimen organizado trasnacional, y otras dinámicas de naturaleza delictivas, llevando en algunos casos a la militarización de la seguridad y al policiamiento de las FFAA (Eissa 2015). Esta agenda entraba en plena contradicción con los principios doctrinarios de la defensa argentina establecidos en los años ochenta, que disponían una estricta separación funcional entre seguridad y defensa, tal como fue plasmado en las leyes de Defensa Nacional y de Seguridad Interior.

Otro dato que debe tenerse en cuenta es que por esos años en la región sudamericana llegaron al poder en diversos países gobiernos de izquierda o progresistas, que exhibieron una conducta contestataria a la proyección de poder e influencia estadounidense en la región. A nivel económico, la defunción del Acuerdo de Libre Comercio de las Américas (ALCA) en 2005 fue el más claro ejemplo. A nivel político, se aceleraron los procesos de integración regional sudamericanos.

El escenario brevemente descripto fue el que permitió el nacimiento de la Unión de Naciones Suramericanas (Unasur) en mayo de 2008, sobre la base de experiencias previas de integración como la Comunidad Suramericana de Naciones, el Mercosur y la Comunidad Andina. Tal como 
señala Pablo Celi (2014, 47), "el rol de las regiones en el mundo, con el cambio de las condiciones de hegemonía, abre el espacio para la emergencia del proyecto suramericano. Proyecto que se asienta en un desplazamiento estratégico de la dimensión continental a la dimensión subregional, para construir una perspectiva suramericana con identidad y autonomía estratégica”. La Unasur se erigía así como un nuevo proyecto de integración suramericana, que se organizaba y se iba montando a partir de la dimensión política, para alcanzar otros ámbitos como la salud, la infraestructura, el desarrollo, la energía, la educación, entre otros, sobre la base del pleno respeto a las instituciones democráticas, los derechos humanos, la soberanía, integridad e inviolabilidad territorial de los Estados, la autodeterminación de los pueblos, la solidaridad y la paz.

Este escenario quedó plasmado en el preámbulo del Tratado Constitutivo de la Unasur, al considerar, los países firmantes, que "la integración es un paso decisivo hacia el fortalecimiento del multilateralismo y la vigencia del derecho en las relaciones internacionales para lograr un mundo multipolar, equilibrado y justo en el que prime la igualdad soberana de los Estados y una cultura de paz" (Unasur 2008b).

En diciembre de ese mismo año, la iniciativa brasileña de generar un espacio de diálogo, cooperación e intercambio en materia de defensa dio surgimiento al Consejo de Defensa Suramericano (CDS). A partir de entonces se iniciaba un proceso único en la región, sin antecedentes previos, que iba a constituirse en el ámbito de cooperación de mayor desarrollo dentro del proceso de integración regional.

Tal como señala Comini (2015), la entonces presidente de Argentina, Cristina Fernández, apoyó la conformación de un grupo de trabajo con el mandato de acordar un estatuto para el Consejo de Defensa. El autor afirma que en las negociaciones de ese grupo de trabajo, la postura que asumió Argentina impactó sobre el perfil que terminó adoptando ese organismo, que inicialmente se disputaba entre dos proyectos, el brasileño de conformar un órgano consultivo, o el venezolano de avanzar en la integración de FFAA sudamericanas, al estilo Otan.

Ugarte (2010) señala que inicialmente, el CDS había despertado altas expectativas que inadecuadamente llevaban a pensarlo como una alianza militar, en tanto sólo se constituía como un mecanismo de cooperación en defensa. Las divergencias existentes en conceptualizaciones sobre seguridad y defensa y en materia de legislación entre sus miembros aparecían como uno de los principales desafíos que podían afectar su desarrollo y consolidación.

Argentina notó tempranamente este aspecto: en enero de 2010 durante la reunión de la Instancia Ejecutiva en Manta, en la cual se discutieron 
los Procedimientos de Aplicación de Medidas de Confianza y Seguridad, el país planteó que muchos asuntos y responsabilidades derivadas al CDS debían encontrar un ámbito exclusivo dentro de la Unasur, toda vez que las cuestiones vinculadas con temas de seguridad pública resultaban ajenas al mandato específico otorgado al CDS. ${ }^{4}$

Esta postura fue ampliamente debatida -presentando particularmente objeciones Colombia- y luego consensuada por los países, quedando plasmada en la II Reunión Ordinaria del CDS. En la Declaración de Guayaquil, de mayo de 2010, se le remitió al Consejo de Ministros de Relaciones Exteriores lo acordado sobre las medidas en el ámbito de la Seguridad -relacionadas con tráfico de armas, acción de grupos armados al margen de la ley y lucha contra el terrorismo- que debían ser abordadas por los órganos e instancias eventuales competentes para ello.

Aparecía así una cuestión para nada menor y fundamental para asegurar el desarrollo de este organismo regional: la necesidad de construir una visión colectiva en materia de Defensa. Para ello, la generación de un pensamiento estratégico regional se tornó un objetivo sine qua non. Particularmente en este aspecto, la Argentina asumió una posición de liderazgo.

En enero de 2009 en Santiago de Chile, en el marco de la Primera Reunión de Viceministros de Defensa del CDS se acordó crear un centro de estudios estratégicos, iniciativa que quedó bajo la responsabilidad de Argentina, país que hizo entrega de un borrador denominado "Proyecto de Constitución del Centro Suramericano de Estudios Estratégicos para la Defensa” (Chile 2009, 135). Tal como señala Braslavsky, "esta contribución de la Argentina se da en el marco del respaldo entusiasta que la cartera que conduce Garré - a tono con los lineamientos de política exterior del gobierno de Cristina Kirchner - le dio a la iniciativa brasileña de crear el Consejo de Defensa Sudamericano" (Braslavsky 2009).

En marzo de 2009, se le otorgó la responsabilidad de dirigir un grupo de trabajo para elaborar la propuesta de estatuto para la creación del Centro de Estudios Estratégicos de la Defensa (CEED-CDS), en el marco del Plan de Acción 2009/2010. Con la Declaración de Guayaquil de mayo de ese año, se aprueba su Estatuto y dos años después, se establece su sede permanente en Buenos Aires, asumiendo como director el argentino Alfredo Forti (Braslavsky 2009).

El CEED-CDS se constituyó así como una instancia regional dedicada al análisis, desarrollo de estudios y asesoramiento en materia de defensa con el objetivo de avanzar en el desarrollo de un pensamiento estratégico sudamericano y la identificación de los intereses regionales en materia de defensa y seguridad regional e internacional, tareas que permitirían "acelerar el debate doctrinario en la región” (Braslavsky 2009). 
Entre las líneas de trabajo e investigación que el CEED-CDS ha desarrollado, se destacan:

- El Observatorio Suramericano de Defensa, que recopila y publica en forma mensual los principales datos y noticias comunicados por los Ministerios de Defensa de los países de la UNASUR.

- El Registro Suramericano de Gastos en Defensa, que permite medir las erogaciones en materia de defensa realizadas por los países miembros, de acuerdo a un instrumento técnico diseñado por el propio CDS, en el marco de las medidas de confianza mutua acordadas en el Plan de Acción del organismo regional.

- Relevamiento de datos y elaboración de informes sobre política de género en los países miembros.

- Registro Suramericano de Inventarios Militares, dirigido a transparentar el inventario de las capacidades militares de los países integrantes de la UNASUR en forma periódica.

- Desarrollo de estudios de prospectiva y estrategia, como "Suramérica 2025 " referido a la defensa de los recursos naturales estratégicos en la región y publicaciones varias como la relativa a la "Institucionalidad de la Defensa en Suramérica”.

- Elaboración de informes e investigaciones varios a requerimiento de los Ministros de defensa de los países miembros del CDS.

Con relación a este último punto, es importante remarcar la labor realizada por el CEED en el marco de la solicitud efectuada en la Reunión Extraordinaria del Consejo de Ministros de Relaciones Exteriores celebrada en Buenos Aires, el 24 de agosto de 2011, en la cual se lo convocó para efectuar estudios sobre la cuestión del crimen organizado trasnacional y otras nuevas amenazas a la seguridad regional, como insumo para una futura reunión de Ministros de Defensa, Justicia e Interior donde se analizarían esos temas.

Como parte del estudio se elaboró el "Informe Preliminar del CEED al CDS acerca de los términos de referencia para los conceptos de seguridad y defensa en la región suramericana”. Este documento argumentó que "la conducción y gestión de las actividades de Seguridad Pública tiende a situar la responsabilidad primaria en ministerios específicos $u$ otras instancias especializadas, distintas de la Defensa Nacional” y señaló que "la seguridad pública, más allá de las diversas denominaciones que puede adoptar, por ejemplo seguridad interior o seguridad ciudadana, está relacionada con la paz social, la estabilidad institucional del Estado, el control del orden público y las garantías de derechos civiles, políti- 
cos, económicos, sociales y culturales, que varían de acuerdo al enfoque político y los objetivos de desarrollo de cada Estado, en relación con los cuales se formulan sus objetivos y percepciones de riesgos y amenazas" (CEED 2012a, 4).

Complementariamente, otro estudio requerido al CEED sobre el crimen organizado transnacional, aseveró que los fenómenos que se presentan asociados a este asunto tales como el narcotráfico, el terrorismo, el tráfico de personas y órganos, el lavado de dinero, los delitos informáticos, el tráfico de bienes y recursos ambientales, el secuestro y acciones provenientes de grupos armados al margen de la ley constituyen fenómenos delictivos asociados a distintos tipos de violencia y configuran problemas de seguridad multifacéticos" (CEED, 2012b, 6).

Claramente, el documento expresa que las distintas modalidades de nuevas amenazas constituyen manifestaciones de criminalidad organizada que adquieren, en diversas ocasiones, dimensiones trasnacionales. En todo caso, pese a la transnacionalidad de su accionar, son concebidas como delitos y por tanto, competencia de las instituciones del Estado responsables de la lucha contra el crimen: Ministerios del Interior, Seguridad Pública, o como éstos se denominen, siendo el instrumento policíaco el responsable de conjurarlo.

Así, el CEED señala que "en la mayoría de los países de la región se enfrenta al crimen organizado y a los delitos transnacionales como una problemática de seguridad pública, abordada, según sus competencias específicas, por Ministerios de Seguridad Pública, Justicia, Interior; con responsabilidades operativas y medios de combate de distinta naturaleza. Ante situaciones de excepción que implican la superación de las capacidades de los sistemas de seguridad pública, la legislación de la mayoría de los Estados prevé el apoyo subsidiario de las FFAA a dichos sistemas de seguridad pública" (CEED, 2012b, 8-9).

De manera conclusiva, el CEED-CDS recomendó entonces la creación de una instancia de cooperación regional "que atienda todos los delitos transnacionales que afectan a la seguridad pública de nuestros países" (2012b, 11).

Fruto de ello, el 30 de noviembre de 2012 se creó, en ocasión de la VI Reunión Ordinaria del Consejo de Jefas y Jefes de Estado y de Gobierno, en Lima, el Consejo Suramericano en Materia de Seguridad Ciudadana, Justicia y Coordinación de Acciones contra la Delincuencia Organizada Transnacional.

De esta manera, el problema de la criminalidad organizada trasnacional quedó fuera de la competencia del CDS, y fue un nuevo consejo regional, el encargado de asumir dicho desafío. 
En conclusión, el CEED tuvo un rol fundamental en el desarrollo institucional del Consejo Suramericano en Materia de Seguridad Ciudadana, Justicia y Coordinación de Acciones contra la Delincuencia Organizada Transnacional, que, bajo la dirección argentina, logró no sólo trasladar, sino también apuntalar en el organismo regional la estricta separación entre Defensa Nacional y Seguridad Interior, uno de los principios liminares de su política defensa (Eissa 2013). ${ }^{5}$

Sobre este aspecto, es interesante citar al ecuatoriano Pablo Celi, quien sostiene que "la Unasur tuvo un acierto al respecto, que fue diferenciar en su estructura, la cooperación en defensa de la cooperación en seguridad pública y lucha contra el crimen organizado. Este fue el primer estudio que realizó el Centro de Estudios Estratégicos de la Defensa (CEED). El mismo fue recogido y aprobado por los Ministros y, posteriormente, por los Presidentes y dio paso a la creación en Unasur de un Consejo de Seguridad Pública diferenciado del Consejo de Defensa Suramericano. Este ha sido un importantísimo avance en doctrina e institucionalidad de cooperación multilateral” (Celi 2014, 50).

\section{CONSIDERACIONES FINALES}

La diferenciación entre seguridad y defensa es un tema que en Argentina, desde la recuperación de la democracia, ha sido objeto de múltiples debates hasta nuestros días. El establecimiento de esta diferenciación en la Ley de Defensa Nacional $n^{\circ} 23.554$ no impidió que posteriormente fuera objeto de discusión por parte de diversos actores que pretendían correr los límites entre ambas esferas para facilitar la incorporación de la agenda de las nuevas amenazas promovida por Washington. Si bien la reglamentación de esa ley en 2006 puso un corset a esos intentos, sigue presentando detractores al día de hoy.

El mismo debate se ha presentado en el seno del CDS. La importancia de construir un pensamiento estratégico regional que pudiera solidificar las bases del organismo regional a partir de las doctrinas y visiones estratégicas que posee cada país miembro, fue una tarea que se encomendó al CEED, bajo la dirección argentina.

Argentina ha tenido desde la creación del CDS un rol protagónico en aspectos clave, como en adiestramiento militar a través del desarrollo del ejercicio Unasur. ${ }^{6}$ Pero fue, tal vez, su contribución a la construcción de una identidad estratégica suramericana en materia de defensa el papel más importante que podía asumir. Dicho papel le permitió moldear la agenda de cooperación, la puesta en común de políticas y conceptos sobre los que se asienta la actividad del CDS. En otras palabras, contribuir a un lenguaje 
común sobre el cual ir asumiendo los desafíos de avanzar en el camino de la cooperación en defensa, indispensable para cimentar cualquier proyecto de construcción de confianza y seguridad para el fortalecimiento y profundización de la zona de paz sudamericana.

En tal sentido, el CDS ha constituido para la Argentina una plataforma de proyección de su doctrina de defensa, sobre la base de la cual confluye la construcción de la identidad estratégica suramericana y de los intereses regionales, lo que evidencia a la vez la capacidad de proyección del denominado "poder blando", o en palabras de Comini $(2015,111)$ "liderazgo de pensamiento" que asumió el país en todo este proceso.

En este aspecto, coincido ampliamente con el autor, cuando plantea que la participación argentina fue fundamental para evitar que sobre el paraguas del CDS "recayeran temáticas que para el país forman parte de la órbita de la seguridad interior, tales como narcotráfico o el terrorismo", lo que permitió concebir al "Consejo como un espacio a partir del cual proyectar su propia concepción sobre la Defensa Nacional y, de esa forma, influir sobre la definición de los ejes prioritarios de la agenda regional en la materia. De alguna forma, el Consejo pasó a ser asumido como una plataforma para actuar de contrapeso a la tendencia manifiesta en varios países sudamericanos de policialización de las FFAA", señala Comini $(2015,125)$.

Por último, la implementación del control civil a través de un proceso de institucionalización de la conducción de la política de defensa, que se desarrolló fundamentalmente a partir del año 2006,facilitó el empleo de la diplomacia de la defensa como una herramienta de gestión de política pública que le permitió a Argentina, por una parte, afianzar un rol protagónico en el Consejo de Defensa Suramericano, y por otro, orientar la diplomacia militar en función de los objetivos políticos, contribuyendo así de manera positiva a la política exterior de la nación.

\section{REFERENCIAS}

Arancibia Morales, Diego. 2013. Diplomacia de la Defensa: Una Aproximación Conceptual. Santiago de Chile: Academia Nacional de Estudios Políticos y Estratégicos. (Serie Artículos Académicos, 6).

Argentina. 2007. Decreto n. 788/07. Boletín Oficial de la República Argentina, Buenos Aires, 25 de junio de 2007.

Argentina. 2007. Decreto n. 1. 729/07. Boletín Oficial de la República Argentina, Buenos Aires, 30 de noviembre de 2007. 
Argentina. Ministerio de Defensa. 2010. Libro Blanco de la República Argentina. Buenos Aires: Ministerio de Defensa.

Argentina. 2013. Decreto n. 636/13. Boletín Oficial de la República Argentina, Buenos Aires, 31 de mayo de 2013.

Argentina. 2014. Decreto n. 1956/14. Boletín Oficial de la República Argentina, Buenos Aires, 28 de octubre de 2014.

Argentina. Ministerio de Defensa. 2015a. Libro Blanco de la República Argentina. Buenos Aires: Ministerio de Defensa.

Argentina. Ministerio de Defensa. 2015b. Misiones y Funciones. Buenos Aires: Ministerio de Defensa. Disponible en:<https://mindef. gov. ar $>$. Fecha de consulta: 18 oct. 2015 .

Argentina. 2015c. Decreto n. 2645/14. Boletín Oficial de la República Argentina, Buenos Aires, 19 de enero de 2015.

Azuara Blanco, Ignacio. 2009. Diplomacia de Defensa. Boletín de Información, Madrid, 308, 75-121.

Braslavsky, Guido. 2009. Argentina Apuesta Debatir la Doctrina Militar de la Región. Clarín, 10 de marzo.

Caviasca, Guillermo. 2015. La actuación del General Jorge Carcagno en la X Conferencia de Ejércitos Americanos en Caracas. Significado, Antecedentes y Debates. Buenos Aires: Escuela de Defensa Nacional. (Serie Documentos de Trabajo, 29).

Celi, Pablo. 2014. Análisis de la Dinámica del Escenario Regional. In Argentina. Ministerio de Defensa. Ciclo de Debates Preparatorios Libro Blanco de la Defensa. Buenos Aires: Ministerio de Defensa. 46-52.

CEED. 2012a. Informe Preliminar del CEED al Consejo de Defensa Suramericano acerca de los términos de referencia para los conceptos de seguridad y defensa en la región suramericana. [En línea]. Centro de Estudios Estratégicos de la Defensa. Disponible en: <http://www. ceedcds. org. ar/Espanol/O9-Downloads/INFORME_ CONCEPTOS_SEG_DEF.pdf>.Fecha de consulta: 11 nov. 2016.

CEED. 2012b. Informe del Centro de Estudios Estratégicos de Defensa del Consejo de Defensa Suramericano, Acerca del Crimen Organizado Transnacional y Otras Nuevas Amenazas a la Seguridad Regional. [En línea]. Centro de Estudios Estratégicos de la Defensa. Disponible en: <http://www. ceedcds. org. ar/Espanol/o9Downloads/CRIMEN_ORGANIZADO. pdf>. Fecha de consulta: 11 nov. 2016. 
Chile. Ministerio de Defensa Nacional de Chile. 2009. El Consejo de Defensa Suramericano. Crónica de su Gestación. Santiago de Chile: Alerce Talleres Gráficos.

Cottey, Andrew; Foster, Anthony. 2004. Reshaping Defence Diplomacy: New Roles for Military Cooperation and Assistance. Oxford: Oxford University Press.

Comini, Nicolás. 2015. El Origen del Consejo Suramericano de Defensa. Modelos en Pugna desde una Perspectiva Argentina. Revista de Estudios en Seguridad Internacional,1,2, 109-135.

Dodd, Tom; Oakes, Mark. 1998. The Strategic Defence Review White Paper. Research Paper 98/91. Londres: House of Commons Library.

Eissa, Sergio. 2013. Política Exterior y Política de Defensa en Argentina: Dos Caras de la Misma Moneda. Perspectivas de Políticas Públicas, 3, 5, 171-191.

Eissa, Sergio. 2015. ¿La Irrelevancia de los Estados Unidos la Política de Defensa Argentina? (1983-2010). Buenos Aires: Arte \& Parte.

Frenkel, Alejandro. 2016. Entre Promesas y Realidades. La Unasur y la Creación de la Escuela Suramericana de Defensa. Relaciones Internacionales, 31, 33-52.

España. Ministerio de Defensa. 2011. Plan de Diplomacia de Defensa. Madrid: Ministerio de Defensa.

Gastaldi, Sol. 2013. Repensando Modelos de Control Civil para las Democracias Latinoamericanas. Un Aporte desde la Teoría de la Agencia. Buenos Aires: Escuela de Defensa Nacional. (Serie Documentos de Trabajo, 11).

Navarro Meza, Miguel. 2009. Política Exterior y Política de Defensa. Una Cercanía Esquiva. Política y Estrategia, 114, 54-116.

Sain, Marcelo. 2010. Los Votos y las Botas. Estudios sobre la Defensa Nacional y las Relaciones Civil-Militares en la Democracia Argentina. Buenos Aires: Prometeo.

Swistek, Göran. 2012. The Nexus between Public Diplomacy and Military Diplomacy in Foreign Affairs and Defence Policy. Connections, XI, 2, 79-86.

Ugarte, José Manuel. 2010. El Consejo de Defensa Suramericano, Balance y Perspectivas. In Mathieu, Hans; Niño Guarnizo, Catalina. (Ed. ). Seguridad Regional en América Latina y el Caribe. Anuario 2010. Bogotá: Friedrich Ebert Stiftung. 25-42.

Unasur. 2008a. Estatuto para el Establecimiento del Consejo de Defensa Suramericano de la UNASUR. Salvador. 
Unasur. 2008b. Tratado Constitutivo de la Unión de Naciones Suramericanas. Brasilia.

Winger, Gregory. 2014. The Velvet Gauntlet: A Theory of Defense Diplomacy. In Lisiak, Agata; Smolenski, Natalie. (Ed. ). What do ideas do?. [En línea]. Viena, IWM Junior Visiting Fellows' Conferences, 33 Disponible en $<$ http://www. iwm. at/ publications/visiting-fellows-conferences/vol-33/>. Fecha de consulta: 21 Abr. 15 


\section{NOTAS}

1. Tomaremos el término de gobierno civil de la defensa desarrollado por Marcelo Sain (2010, 35-36), quien lo conceptualiza como el patrón o modalidad que adquieren las relaciones civiles-militares bajo un régimen democrático cuando se verifica el ejercicio efectivo del gobierno político-institucional sobre las FFAA y la subordinación efectiva de las FFAA a las autoridades gubernamentales, modalidad que supone "el ejercicio efectivo y competente de la conducción gubernamental -ejecutiva y legislativa- sobre las FFAA en todo lo atinente al establecimiento de las bases legales, orgánicas y funcionales de la defensa nacional; la estructuración y gestión del sistema institucional de gobierno de la defensa nacional y las FFAA; la fijación y el control del cumplimiento de las misiones y funciones institucionales específicas de esas fuerzas; la formulación y gestión de la estructura orgánica, funcional y doctrinal de las mismas; y el tratamiento y abordaje de todas aquellas cuestiones y actividades derivadas del funcionamiento político-institucional de las FFAA o que supongan la vinculación o intervención institucional de éstas".

2. Las otras dos áreas de trabajo son: Comisiones al Exterior y Agregadurías; y Cooperación Internacional de Defensa.

3. Un ejemplo de ello fue la designación que recibió Argentina por parte de los EE.UU. de "non-NATO major ally".

4. El CDS se erigió con los objeticos de consolidar una zona de paz suramericana; construir una visión común en materia de defensa; articular posiciones regionales en foros multilaterales sobre defensa; cooperar regionalmente en materia de defensa; apoyar acciones de desminado, prevención, mitigación y asistencia a víctimas de desastres naturales (Unasur 2008a).

5. Eissa $(2013,172)$ sostiene que la política de defensa argentina se asienta sobre tres principios liminares: la supresión de las hipótesis de conflicto con los países vecinos que requieran la utilización de las FFAA; la separación entre defensa nacional y seguridad interior; y el gobierno civil de la política de defensa.

6. Entre otras responsabilidades que asumió Argentina, se destacó la de integrar el grupo de trabajo que dio origen a la Escuela Suramericana de Defensa (Esude) y el dictado del Programa Suramericano de Formación en Defensa entre 2012 y 2015. Ver Frenkel (2016). 


\section{RESUMEN}

El presente artículo analiza la dimensión internacional de la política de defensa argentina (2008-2015) para concluir que el Consejo de Defensa Suramericano representa una plataforma para la diplomacia del país, que le permite proyectar regionalmente su doctrina de defensa y contribuir de manera positiva a la política exterior.

Palabras clave: Consejo de Defensa Suramericano; Diplomacia de defensa; Diplomacia militar; Gobierno civil de las FFAA.

\section{ABSTRACT}

This paper inquires about the international dimension of Argentinean defense policy (2008-2015) to conclude that the South American Defense Council represents a platform for defense diplomacy, which allows the country to project regionally its defense doctrine and to contribute positively to the foreign policy.

Keywords: South American Defense Council; Defense diplomacy; Military diplomacy; Civilian government of the armed forces. 